\title{
An exact solution for the propagation of cylindrical shock waves in a rotational axisymmetric non- ideal gas with axial magnetic field and radiative heat flux
}

\author{
G. Nath ${ }^{*}$, P.K. Sahu ${ }^{2}$, S. Chaurasia ${ }^{3}$ \\ ${ }^{1}$ Department of Mathematics, Motilal Nehru National Institute of Technology Allahabad, Allahabad-211004, India \\ ${ }^{2}$ Department of Mathematics, SPM College, Sitapur-497111, Sarguja University, India \\ ${ }^{3}$ Mechanical Engineering Department, MMMUT Gorakhpur-273010, India
}

Corresponding Author Email: gnath@mnnit.ac.in

https://doi.org/10.18280/mmc_b.870404

Received: 20 May 2018

Accepted: 15 September 2018

\section{Keywords:}

shock waves, similarity solution, rotating medium, non-ideal gas, radiation heat flux, magnetogasdynamics

\begin{abstract}
Propagation of cylindrical shock wave in a rotational axisymmetric non-ideal gas with axial magnetic field, radiation heat flux and the components of vorticity vectors are investigated. The axial magnetic field and the fluid velocity in the ambient medium are assumed to vary and obey the power laws. An exact similarity solution is obtained. The total energy of the shock wave is not constant but increases with time. The effects of variation of parameter of non-idealness of the gas, the Alfven-Mach number and the adiabatic exponent of the gas are investigated. It is shown that an increase in the non-idealness of the gas or the ratio of specific heats of the gas or strength of initial magnetic field decreases the shock strength but increases the shock velocity. Further it is observed an increase in the value of parameter of non-idealness of the gas and adiabatic exponent of the gas have same behavior on the flow variables and the shock strength.
\end{abstract}

\section{INTRODUCTION}

The problem of propagation of magnetogasdynamics shock waves in a rotating interplanetary atmosphere assumes special significance in the study of astrophysical phenomena. The experimental studies and astrophysical observations show that due to rotation of the planets and stars the outer atmosphere of the planets and stars rotates. Macroscopic motion with supersonic speed occurs in an interplanetary atmosphere with rotation and shock waves are generated. Thus, the rotation of planets or stars considerably affects the process taking place in their outer layers, therefore question connected with the explosions in rotating gas atmospheres are of definite astrophysical interest.

Chaturani [1] studied the propagation of cylindrical shock waves through a gas with solid body rotation and obtained solutions using the similarity method adopted by Sakurai [2]. Nath et al. [3] derived similarity solutions for the flow behind the shock waves propagating in a non-uniform rotating interplanetary atmosphere with increasing energy. Gangualy and Jana [4] studied a theoretical model for the propagation of strong spherical shock waves in a self-gravitating atmosphere with a radiation flux in the presence of a magnetic field. They also considered that the medium behind the shock rotated, but neglected rotation of the undisturbed medium. In all the works mentioned, the medium is taken to be a gas satisfying the equation of state of a perfect gas.

In recent years the problems of radiative energy transfer in fluids have received extensive attention as a consequence of the increasing speed of bodies through the atmosphere and the very high temperatures attained by gases in motion. The influence of radiation on a shock wave and on the flow-field behind the shock front has always been of great interest, for instance, in the field of nuclear power and space research.
Similarity solutions in radiation-gas-dynamics have been given by Marshak [5], Elliott [6] and Wang [7] in which the flow is headed by a shock wave. Elliot [6] considered the explosion problem, solved earlier by Taylor [8] by introducing the radiation flux in its diffusion approximation. Wang [7] has discussed 'piston problem' with radiation energy transfer in the thick limit and thin limit, and also the general case with the idealized 'two direction' approximation. Ashraf and Sachdev [9] did not explicitly use the radiation transfer equation but evaluated the radiation flux from the conservation equations. Their solutions, therefore, hold without any restrictions imposed on optical properties of the medium. Verma and Vishwakarma [10] extended the problem treated by Ashraf and Sachdev [9] to take into account the effects of transverse magnetic field.

Because of high pressure and density that usually occur behind a shock wave, produced by an explosion, the belief that the gas is perfect is no longer valid. In recent years, several studied are performed regarding the problem of shock waves in non-ideal gases, specifically, by Anisimov and Spiner [11], Ranga Rao and Purohit [12], Vishwakarma and Nath [13-14], Nath [15] and many others. The popular alternative to the perfect gas might be a simplified van der Waals model. Wu and Roberts [16], Roberts and $\mathrm{Wu}$ [17] adopted this model to discuss the shock wave theory of sonoluminescence.

In the present work, we also adopt this model of a non-ideal gas to obtain a self-similar solution for the flow behind a magnetogasdynamics cylindrical shock wave. Shock wave is assumed to be propagating in a non-ideal gas permeated by an axial magnetic field in a rotational axisymmetric flow of a gas with constant density, which has variable azimuthal and axial fluid velocities [18-20]. The fluid velocities and the axial magnetic field in the ambient medium are assumed to vary as some power of the distance from the symmetry axis. Also, the 
angular velocity of rotation of the ambient medium is assumed to be obeying a power law and to be decreasing as the distance from the axis increases. It is expected that such an angular velocity may occur in the atmospheres of rotating planets and stars.

In the present problem we have extended the problem treated by Vishwakarma and Patel [21] by taking into account the axial component of fluid velocity and components of vorticity vectors. Also, we have study the effect of adiabatic exponent of the gas on the flow variables. The exact similarity solutions are obtained with the general shock conditions in place of strong shock conditions and the shock is assumed to be transparent. We have taken the similarity form for energy and radiative heat flux [9] and the 'Product Solutions' of Mc. Vittie [22] is used to evaluate it. We have evaluated radiation flux from conservation equations.

The effects of variation of parameter of non-idealness of the gas, the Alfven-Mach number, and the ratio of specific heats of the gas are investigated. It is shown that an increase in the value of adiabatic exponent of the gas or in the parameter of non-idealness of the gas or the presence of magnetic field have decaying effect on shock strength. An increase in the value of Alfven-Mach number and parameter of non-idealness of the gas have opposite behaviour on pressure and axial magnetic field; whereas they have same behaviour on radial component of fluid velocity, density, radiation heat flux and the azimuthal component of vorticity vector.

\section{FUNDAMENTAL EQUATIONS OF MOTIONS AND BOUNDARY CONDITIONS}

The fundamental equations governing the one-dimensional, unsteady adiabatic and cylindrically symmetric rotational flow of a non-ideal gas in the presence of an axial magnetic field in which the effect of radiation heat flux may be significant, in Eulerian coordinates, can be expressed as [15, 18-20, 23-25]

$$
\frac{\partial \rho}{\partial t}+u \frac{\partial \rho}{\partial r}+\rho \frac{\partial u}{\partial r}+\frac{u \rho}{r}=0,
$$

$\frac{\partial u}{\partial t}+u \frac{\partial u}{\partial r}+\frac{1}{\rho}\left[\frac{\partial p}{\partial r}+\mu h \frac{\partial h}{\partial r}\right]-\frac{v^{2}}{r}=0$

$\frac{\partial v}{\partial t}+u \frac{\partial v}{\partial r}+\frac{u v}{r}=0$

$\frac{\partial w}{\partial t}+u \frac{\partial w}{\partial r}=0$

$$
\frac{\partial w}{\partial t}+u \frac{\partial w}{\partial r}=0
$$

$\frac{\partial h}{\partial t}+u \frac{\partial h}{\partial r}+h \frac{\partial u}{\partial r}+\frac{h u}{r}=0$

$\frac{\partial e}{\partial t}+u \frac{\partial e}{\partial r}-\frac{p}{\rho^{2}}\left[\frac{\partial \rho}{\partial t}+u \frac{\partial \rho}{\partial r}\right]+\frac{1}{\rho r} \frac{\partial(F r)}{\partial r}=0$,

where $\mathrm{r}$ and $\mathrm{t}$ are the independent space and time coordinates; $u, v$, and $w$ are the radial, azimuthal and axial components of the fluid velocity $\vec{q}$ in the cylindrical coordinates $(r, \theta, \mathrm{z})$; $\rho, \mathrm{p}, e, h$ and $F$ are the density, the pressure, the internal energy per unit mass, the axial magnetic field and the radiation heat flux respectively.
The general figure showing the velocity components in Eulerian coordinate in the case of rotational axisymmetric onedimensional flow is shown in Figure A [19].

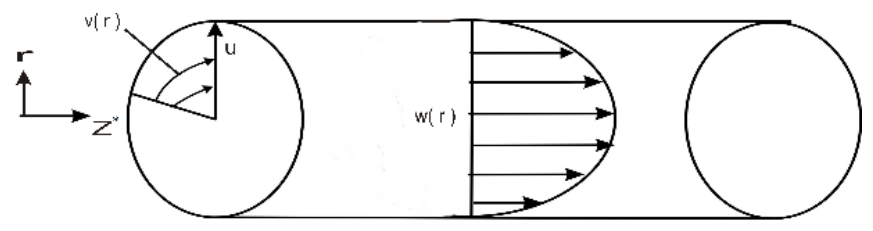

Figure A. Show the directions of velocity vector

The relation between angular velocity of the medium and the azimuthal component of fluid velocity is given by

$$
v=C r
$$

where ' $C$ ' is the angular velocity of the medium at radial distance $r$ from the axis of symmetry. In this case the vorticity vector $\vec{\varsigma}=\frac{1}{2} \operatorname{Curl} \vec{q}$ has the components

$$
\varsigma_{r}=0, \quad \varsigma_{\theta}=-\frac{1}{2} \frac{\partial w}{\partial r}, \quad \varsigma_{z}=\frac{1}{2 r} \frac{\partial}{\partial r}(r v)
$$

The system of equations (1) - (6) should be supplemented with an equation of state. Most of the phenomena associated with shock wave arise in extreme conditions under which the ideal gas is not a sufficiently accurate description. To discover how deviations from the ideal gas can affect the flow behind a shock wave, we adopt a simple model. We assume that the gas obeys a simplified van der Waal equation of state of the form (Nath [15], Wu and Roberts [16], Roberts and Wu [17], Nath and Vishwakarma [26])

$$
p=\frac{\Gamma T}{\left(v^{*}-b\right)}, e=C_{v} T=\frac{p\left(v^{*}-b\right)}{(\gamma-1)},
$$

where $\Gamma$ is the gas constant, $\gamma$ is the ratio of specific heats, $T$ is the temperature of the gas, $v^{*}=1 / \rho$ is the specific volume, $C_{v}$ is the specific heat at constant volume, constant $b$ is the van der Waals excluded volume.

The relation between the shock velocity $W\left(=\frac{d R}{d t}\right)$ and shock radius $R$ is assumed to be given as (Ashraf and Sachdev [9])

$W^{2}=a_{1}^{2} R^{-\lambda}$,

where ' $\boldsymbol{a}_{\mathbf{1}}$ ' and ' $\boldsymbol{\lambda}$ ' are constants.

A diverging cylindrical shock wave is assumed to be propagating outwards from the axis of symmetry in the undisturbed non-ideal gas with constant density in the presence of an axial magnetic field. In order to obtain the similarity solution, it is assumed that initial angular velocity of the medium varies as $C_{0}=C_{a} R^{\delta}$, where $C_{a}$ and $\delta$ are constants. The flow variables immediately ahead of the shock front are

$$
\begin{aligned}
& u=u_{0}=\mathbf{0}, \rho=\rho_{0}=\text { constant, } v=v_{0}=C_{a} R^{\delta+1},(-1< \\
& \delta<0), w=w_{0}=w_{a} R^{\alpha}, h_{0}=h_{a} R^{-\sigma}
\end{aligned}
$$


$p_{0}=-\frac{\mu h_{a}^{2}}{2} R^{-2 \sigma}+\frac{\rho_{0} C_{a}^{2}}{(2 \delta+2)} R^{2 \delta+2}$,

where $w_{a}, h_{a}, \alpha$ and $\sigma$ are constants; $\boldsymbol{\sigma}=-(\boldsymbol{\delta}+\mathbf{1})$, and subscript ' 0 ' refers the conditions immediately ahead of the shock front. The initial pressure $p_{0}$ is positive if $1+\delta>0$.

Ahead of the shock, the components of the vorticity vector, therefore vary as

$\varsigma_{r_{0}}=0, \quad \varsigma_{\theta_{0}}=-\frac{1}{2} \frac{\alpha w_{0}}{R}, \quad \varsigma_{z_{0}}=\frac{1}{2} \frac{(\delta+2) v_{0}}{R}$

We assume the shock surface to be transparent; therefore, the radiation heat flux is continuous across it. Thus, the jump conditions across the magnetogasdynamics shock propagating into rotating non-ideal gas are given by (Vishwakarm and Patel [21], Vishwakarma et al. [25]

$u_{1}=(1-\beta) W, \quad \rho_{1}=\frac{\rho_{0}}{\beta}, \quad p_{1}=L \rho_{0} W^{2}, \quad h_{1}=\frac{h_{0}}{\beta}, \quad F_{1}=F_{0}, \quad v_{1}=v_{0}, \quad w_{1}=w_{0}$,

where $L=\left[(1-\beta)+\frac{1}{\gamma M^{2}}+\frac{M_{A}^{-2}}{2}\left(1-\frac{1}{\beta^{2}}\right)\right], M=\left(\frac{\rho_{0} W^{2}}{\gamma p_{0}}\right)^{\frac{1}{2}}$ is the shock Mach number referred to the frozen speed of sound $\left(\frac{\gamma p_{0}}{\rho_{0}}\right)^{\frac{1}{2}}$ and $M_{A}=\left(\frac{\rho_{0} W^{2}}{\mu h_{0}^{2}}\right)^{\frac{1}{2}}$ is the Alfven- Mach number, and the subscript ' 1 ' denotes the condition immediately behind the shock front. The density ratio $\beta(0<\beta<1)$ across the shock is obtained by the

$\beta^{3}-\beta^{2}\left[\frac{2}{(\gamma+1) M^{2}}+\frac{2 \bar{b}+\gamma\left(1+M_{A}^{-2}\right)-1}{(\gamma+1)}\right]+\beta\left[\frac{\bar{b}+\gamma-2}{(\gamma+1)}\right] M_{A}^{-2}+\frac{\bar{b} M_{A}^{-2}}{(\gamma+1)}=0$,

where $\bar{b}=b \rho_{0}$.

Following Levin and Skopina [18] and Nath [15, 19, 20], we get the jump conditions for the components of vorticity vector across the shock front as

$\varsigma_{\theta_{1}}=\frac{{ }^{\varsigma_{\theta_{0}}}}{\beta} ; \quad \varsigma_{z_{1}}=\frac{\varsigma_{z_{0}}}{\beta}$.

\section{SELF-SIMILARITY TRANSFORMATIONS}

To obtained the similarity solutions, the field variables describing the flow pattern can be written in terms of the dimensionless functions of $\xi$ such that (Vishwakarma et al. [25], Vishwakarm and Patel [21])

$u=W U(\xi), v=W \phi(\xi), w=W V(\xi), \rho=\rho_{0} G(\xi)$,

$p=\rho_{0} W^{2} P(\xi), \sqrt{\mu} h=\sqrt{\rho_{0}} W H(\xi), e=W^{2} E(\xi)$,

$F=\rho_{0} W^{3} \bar{F}(\xi)$,

where $\boldsymbol{U}, \phi, V, G, P P, H, E$, and $\bar{F}$ are the function of $\boldsymbol{\xi}$ only, $\boldsymbol{\xi}=\boldsymbol{r} / \boldsymbol{R}$ is the dimensionless quantity.

For existence of similarity solutions $M$ and $M_{A}$ should be constants, therefore,

$-\lambda=2 \delta+2$, and $\lambda=2 \sigma$
Thus,

$M^{2}=\gamma^{-1}\left[-\frac{1}{2}+\frac{\rho_{0} C_{a}^{2}}{(2 \delta+2) \mu h_{a}^{2}}\right]^{-1} M_{A}^{2}$.

By using similarity transformations from equation (16), equations (1)-(6) can be transformed and simplified to

$$
\begin{aligned}
& (U-\xi) \frac{d G}{d \xi}+G \frac{d U}{d \xi}+\frac{G U}{\xi}=0, \\
& (U-\xi) \frac{d U}{d \xi}-\frac{\lambda U}{2}+\frac{1}{G}\left[\frac{d P}{d \xi}+H \frac{d H}{d \xi}\right]-\frac{\phi^{2}}{\xi}=0, \\
& (U-\xi) \frac{d \phi}{d \xi}+\left(\frac{U}{\xi}-\frac{\lambda}{2}\right) \phi=0, \\
& (U-\xi) \frac{d V}{d \xi}-\frac{\lambda}{2} V=0,
\end{aligned}
$$

$\frac{d U}{d \xi} H+(U-\xi) \frac{d H}{d \xi}+\frac{U H}{\xi}-\frac{\lambda H}{2}=0$,

$(U-\xi) \frac{d E}{d \xi}-\lambda E+\frac{P}{G}\left(\frac{d U}{d \xi}+\frac{U}{\xi}\right)+\frac{1}{G \xi} \frac{d}{d \xi}(\xi \bar{F})=0$.

Using the similarity transformations (16), the shock conditions (13), transforms into

$$
\begin{aligned}
& U(1)=(1-\beta), G(1)=\frac{1}{\beta}, P(1)=L, H(1)=\frac{1}{\beta M_{A}}, \\
& \phi(1)=\sqrt{\frac{\rho_{0}}{\mu}} \frac{C_{a}}{h_{a}} M_{A}^{-1}, V(1)=\sqrt{\frac{\rho_{0}}{\mu}} \frac{w_{a}}{h_{a}} M_{A}^{-1},
\end{aligned}
$$

where $\alpha=(\delta+1)=-\sigma$ was necessary to use to obtain the similarity solution.

We assume the product solution of the 'progressive wave' in the form (c.f. Mc. Vittie [22])

$u=\frac{a(t)}{t} r, \rho=(d+1) f(t) t^{-2 \varepsilon} x^{d-2}, \quad p=\varepsilon^{2} f(t) t^{-2} b_{0}(t) x^{d}$,

$h=\varepsilon f^{\frac{1}{2}}(t) t^{-1} c(t) x^{d / 2}, \quad v=\frac{m(t)}{t} r, w=\frac{g(t)}{t} r$

where $\boldsymbol{x}=\boldsymbol{r} \boldsymbol{t}^{-\varepsilon}, d$ and $\varepsilon$ are constants, and $\boldsymbol{a}, \boldsymbol{f}, \boldsymbol{b}_{\mathbf{0}}$ and $\boldsymbol{c}$ are functions of $t$ that satisfy the following equations:

$$
\begin{aligned}
& a(t)=\frac{\varepsilon d-t \frac{f^{\prime}}{f}}{d}=\frac{1}{2}-\frac{t c^{\prime}}{2 c}=\frac{1}{2}-\frac{t m^{\prime}}{2 m}=1-\frac{t g^{\prime}}{g}, \\
& b_{0}(t)+\frac{\mu}{2} c^{2}(t)=\frac{(d+1)}{d \varepsilon^{2}}\left(a-a^{2}-t a^{\prime}+m^{2}(t)\right) .
\end{aligned}
$$

It can be easily seen that these equations satisfy the equations (1) to (5) identically. After changing this solution to a similarity one, from which it follows that $a$ should be constant $a=\left(\frac{2(1-\beta)}{\lambda+2}\right)$, we apply boundary conditions (25) and obtain the solution of the considered problem 
$U(\xi)=(1-\beta) \xi, \quad G(\xi)=\frac{1}{\beta} \xi^{d-2}, \quad P(\xi)=L \xi^{d}, \quad H(\xi)=\frac{1}{\beta M_{A}} \xi^{d / 2}, \quad l_{r}=0, \quad l_{\theta}=\sqrt{\frac{\rho_{0}}{\mu}} \frac{W_{a} \lambda}{4 h_{a} \beta} M_{A}^{-1}, \quad l_{z}=\sqrt{\frac{\rho_{0}}{\mu}} \frac{C_{a}(2-\lambda)}{4 h_{a} \beta} M_{A}^{-1}$.

$\phi(\xi)=\sqrt{\frac{\rho_{0}}{\mu}} \frac{C_{a}}{h_{a}} M_{A}^{-1} \xi, V(\xi)=\sqrt{\frac{\rho_{0}}{\mu}} \frac{w_{a}}{h_{a}} M_{A}^{-1} \xi$

\section{RESULTS AND DISCUSSION}

Substituting equations (9) - (10) and (29) in equation (24), we evaluate the value of $\bar{F}(\xi)$ as

$\bar{F}(\xi)=\frac{L \xi^{d+1}}{(\gamma-1)(d+2)}[2 \beta+\lambda-2(1-\beta)(\gamma-1)]-\frac{L \bar{b} \xi^{2 d-1}}{2 d(\gamma-1)}\left(d+\frac{\lambda}{\beta}\right)$.

Substituting equations (29) into equations (19) and (20) we obtain

$d=\frac{2}{\beta} \quad$ and $\quad \frac{\rho_{0} C_{a}^{2}}{\mu h_{a}^{2}} M_{A}^{-2}=\beta(1-\beta)(d-1)-\frac{\lambda}{2}(1-\beta)+\frac{\beta d}{\gamma M^{2}}+\frac{M_{A}^{-2}}{2} d \beta$.

From equations (21) - (23) and (29), we obtain

$\lambda=2(1-2 \beta)$ and $\lambda=-2 \beta$

It is obvious that a contradiction arises and therefore it is impossible for the solutions (29) to satisfy (19) - (24), though they can satisfy (19), (20) and (24) (i.e. conservation of mass, linear momentum and energy (c.f. Mc. Vittie [22])). For the existence of solution, we consider the physical conditions of the problem to avoid the contradiction. Since $\lambda<0$ and $0<$ $\beta<1$; therefore for the existence of solution we have taken $\lambda=2(1-2 \beta)$, thus $\beta$ should be greater that $1 / 2$. Then, from (17) we have

$\sigma=(1-2 \beta), \quad \delta=-2(1-\beta)$.

Total energy $E_{T}$ of the flow field behind the shock front is given as

$E_{T}=2 \pi \int_{0}^{R}\left\{\frac{p(1-b \rho)}{(\gamma-1) \rho}+\frac{\mu h^{2}}{2 \rho}+\frac{1}{2}\left(u^{2}+v^{2}+w^{2}\right)\right\} \rho r d r$

Using equations (16) and (29), equation (34) becomes

$E_{T}=2 \pi \rho_{0} a^{2} J R^{2-\lambda}=B t^{\frac{2(2-\lambda)}{2+\lambda}}$,

where

$J=\int_{0}^{1}\left\{\frac{L}{(\gamma-1)}\left(1-\frac{\bar{b}}{\beta} \xi^{d-2}\right)+\frac{1}{2 \beta^{2} M_{A}^{2}}+\frac{(1-\beta)^{2}}{2 \beta}+\frac{\rho_{0}}{\mu} \frac{C_{a}^{2}}{h_{a}^{2}} M_{A}^{-2}+\frac{\rho_{0}}{\mu} \frac{w_{a}^{2}}{h_{a}^{2}} M_{A}^{-2}\right\} \xi^{d+1} d \xi$,

and $B$ is constant. This equation shows that the total energy behind the shock increases with time. Such an increase may be obtained by time-dependent energy release from an explosive material along the symmetry axis.

Applying the similarity transformations (16) on equation (8) and using equations (19) - (24), (29), we obtain the nondimensional components of vorticity vector $l_{r}=\frac{\varsigma_{r}}{\frac{W}{R}}, l_{\theta}=$ $\frac{S_{\theta}}{\frac{W}{R}}, l_{z}=\frac{S_{z}}{\frac{W}{R}}$ in the flow-filed behind the shock as
For the density to remain finite at the center and the radiative heat flux not to be negative anywhere, the inequalities obtained from equations (26) and (30) should hold:

$1 / 2<\beta<1$,

$\frac{\xi^{d+1}}{(\gamma-1)(d+2)}[2 \beta+\lambda-2(1-\beta)(\gamma-1)]-\frac{\bar{b} \xi^{2 d-1}}{2 d(\gamma-1)}\left(d+\frac{\lambda}{\beta}\right)>0$

Inequality (37) is not only a necessary condition for the density to remain finite at the center but must also be satisfied for the existence of a shock wave. We have calculated the values of flow variables from equations (29), (30), (36) - (38) and the results are shown in figures 1,2 and Table 1 . The values of the physical parameters for calculations are taken to be $\gamma=4 / 3,5 / 3 ; M_{A}^{-2}=0.3,0.35$; and $\bar{b}=0,0.05,0.1$; ( $[14$, $15,20,26-33])$. For fully ionized gas $\gamma=5 / 3$ and for relativistic gases $\gamma=4 / 3$. These two values of $\gamma$ mark the most general range seen in stars. Therefore, it is applicable to stellar medium. The above values of $M_{A}^{-2}$ are taken for calculations in the present problem as the effects of magnetic field on the flow-field behind the shock are significant when $M_{A}^{-2} \geq 0.01$ ([29]). The value $\bar{b}=0$ corresponds to the ideal gas case. Our solution is the generalization of the solution obtained by Vishwakarma and Patel [21] by considering the components of vorticity vectors and the axial component of fluid velocity (see Figs. 1 (c, h, i). Also, we have study the effect of adiabatic exponent of the gas on the flow variables.

The obtained solutions show that the component of fluid velocity, density, pressure, axial magnetic field and radiation heat flux tend to zero as the symmetry axis is approached. Also, the values of all this physical quantities decrease from the highest at the shock to zero at the symmetry axis, except for the radiation heat flux in the case of $\gamma=5 / 3$ and $\bar{b}=0.1$. For $\gamma=5 / 3, \bar{b}=0.1$ the radiation heat flux first increases, attains a maximum, and then decreases and tends to zero as the symmetry axis is approached. But the azimuthal and axial components of vorticity vector remain constant throughout the flow field.

From Table 1 and Figs. 1 and 2, it is found that the effects of an increase in the value of $M_{A}^{-2}$ (i.e., in the strength of the initial magnetic field) are

(i) to increase significantly the value of $\beta$ (see Table 1), i.e., decreases the shock strength;

(ii) to decrease the flow variables $U(\xi), P(\xi), F(\xi)$ and $l_{\theta}$; whereas to increase the flow variables $\phi(\xi), V(\xi), H(\xi)$ and $l_{z}$ at any point in the flow field behind the shock (see Figures. 2 (a-c, e-i));

(iii) the density $G(\xi)$ decrease near shock but increase near inner boundary surface (see Fig.1(d));

(iv) increases the shock velocity $W / a_{1}$ (see Figure. 1).

The effects of an increase in the value of the parameter of non-idealness of the gas $\bar{b}$ are

(i) to increase the value of $\beta$ (see Table 1), i.e., decreases the shock strength; 
(ii) to decrease the flow variables $U(\xi), H(\xi), F(\xi)$ and $l_{\theta}$; whereas to increase the pressure $P(\xi)$ at any point in the flow field behind the shock (see Figs. 1 (a, e, f-h));

(iii) the density $G(\xi)$ decreases near shock but increases near inner boundary surface (see Fig. 1(d)); (iv) the flow variables $\phi(\xi), V(\xi)$ and $l_{z}$ remain unaffected (see Fig. 1 (b, c, i));

(v) increases the shock velocity $W / a_{1}$ (see Figure. 1 and table 1).

Table 1. Variations of the density ratio $\beta$ across the shock front, indices of shock velocity $\lambda$, total energy $2(2-\lambda) /(2+\lambda)$ and initial angular velocity indices $\delta$ for different values of $M_{A}^{-2}, \gamma$ and $\bar{b}$.

\begin{tabular}{ccccccc}
\hline$M_{A}^{-2}$ & $\gamma$ & $\bar{b}$ & $\beta$ & $\lambda=2(1-2 \beta)$ & $2(2-\lambda) /(2+\lambda)$ & $\delta=-2(1-\beta)$ \\
\hline \multirow{6}{*}{0.3} & & 0 & 0.5296 & -0.1187 & 2.2524 & -0.9406 \\
& $4 / 3$ & 0.05 & 0.5362 & -0.1448 & 2.3123 & -0.9275 \\
& & 0.1 & 0.5440 & -0.1762 & 2.3864 & -0.9119 \\
& $5 / 3$ & 0.05 & 0.5522 & -0.2091 & 2.4670 & -0.8954 \\
& & 0.1 & 0.5708 & -0.2433 & 2.5540 & -0.8783 \\
& & 0 & 0.5714 & -0.2833 & 2.6602 & -0.8583 \\
0.35 & 0.05 & 0.5770 & -0.3082 & 2.6666 & -0.8571 \\
& & 0.1 & 0.5837 & -0.3349 & 2.7287 & -0.8458 \\
& $5 / 3$ & 0.05 & 0.5898 & -0.3592 & 2.8046 & -0.8325 \\
& & 0.1 & 0.6054 & -0.4216 & 2.8756 & -0.8203 \\
& & & & & 2.9628 & -0.8059 \\
\end{tabular}
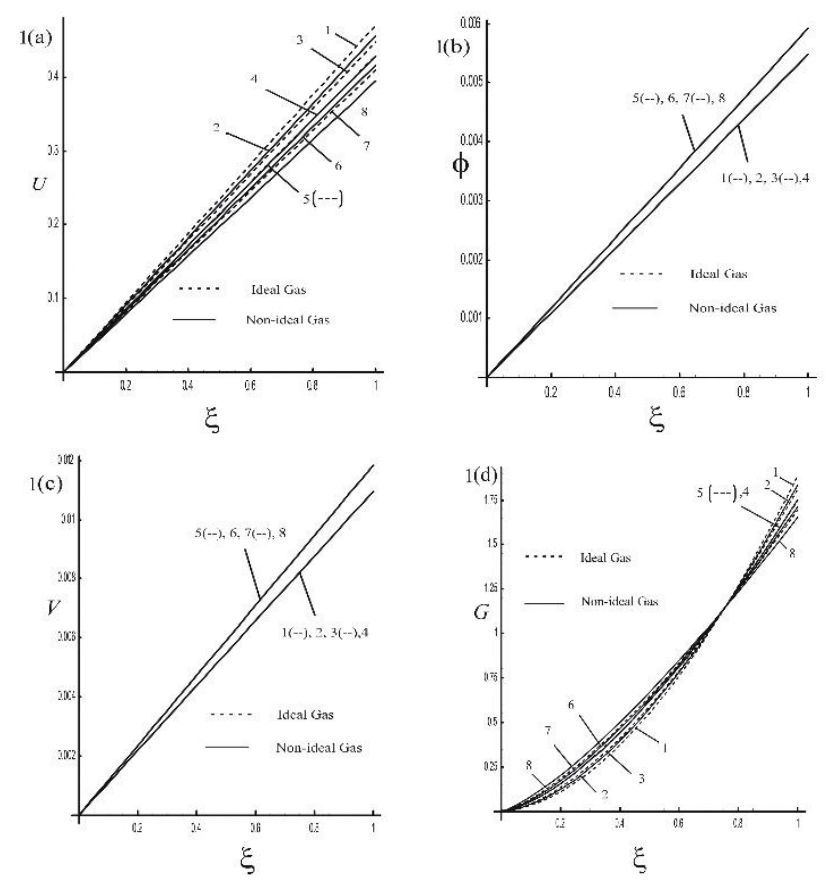

Figure 1. Variation of the flow variables with the distance in the region behind the shock front

(a) radial component of fluid velocity $U(\xi)$, (b) azimuthal component of fluid velocity $\phi(\xi)$, (c) axial component of fluid velocity $V(\xi)$, (d) density $G(\xi)$, (e) pressure $P(\xi)$, (f) axial magnetic field $H(\xi)$, (g) radiation flux $F(\xi)$, (h) nondimensional azimuthal component of vorticity vector $l_{\theta}$, (i) non-dimensional axial component of vorticity vector $\mathrm{l}_{\mathrm{z}}: 1$. $M_{A}^{-2}=0.3, \gamma=4 / 3, \bar{b}=0 ; 2 . M_{A}^{-2}=0.3, \gamma=4 / 3, \bar{b}=0.1$; 3. $\mathrm{M}_{\mathrm{A}}^{-2}=0, \gamma=5 / 3, \overline{\mathrm{b}}=0 ; 4 . \mathrm{M}_{\mathrm{A}}^{-2}=0.3, \gamma=5 / 3, \overline{\mathrm{b}}=0.1$; 5. $\mathrm{M}_{\mathrm{A}}^{-2}=0.35, \gamma=4 / 3, \overline{\mathrm{b}}=0 ; 6 . \mathrm{M}_{\mathrm{A}}^{-2}=0.35, \gamma=4 / 3, \overline{\mathrm{b}}=0.1$; 7. $\mathrm{M}_{\mathrm{A}}^{-2}=0.35, \gamma=5 / 3, \overline{\mathrm{b}}=0 ; 8 . \mathrm{M}_{\mathrm{A}}^{-2}=0.35, \gamma=5 / 3, \overline{\mathrm{b}}=0.1$.
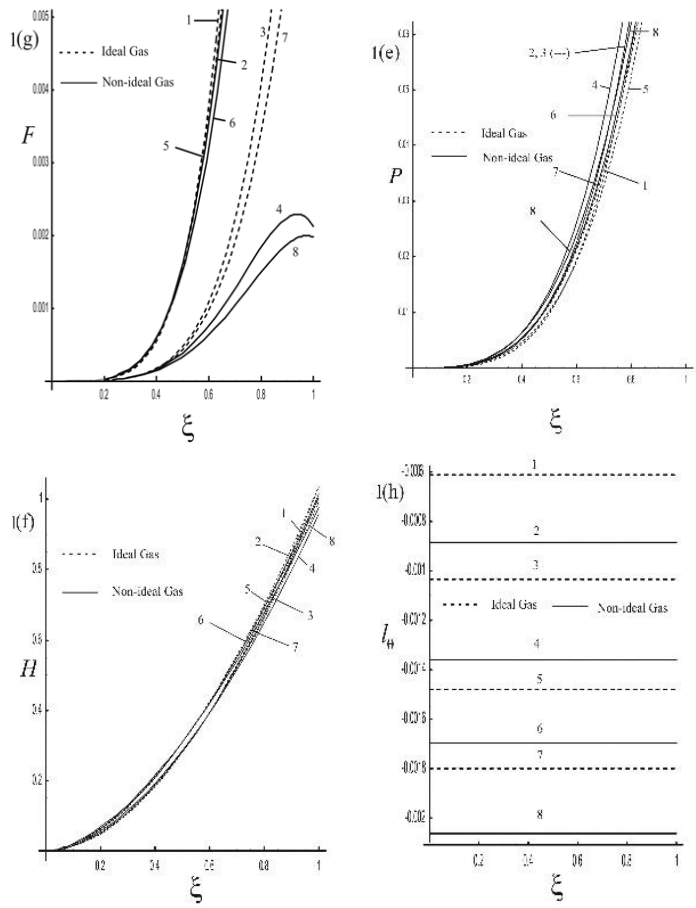

Figure 1. (Continued)

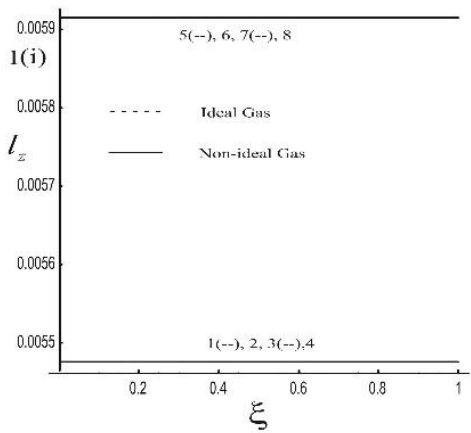

Figure 1. (Continued) 


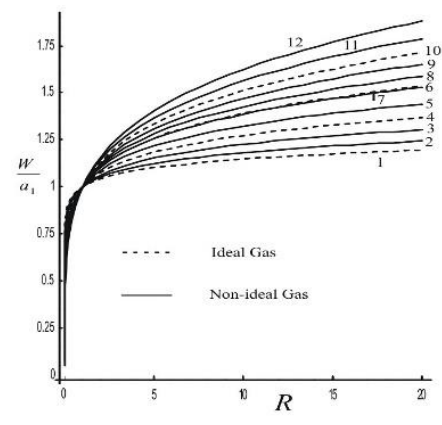

Figure 2. Variation of the shock velocity with the shock radius

1. $\mathrm{M}_{\mathrm{A}}^{-2}=0.3, \gamma=4 / 3, \overline{\mathrm{b}}=0 ; 2 . \mathrm{M}_{\mathrm{A}}^{-2}=0.3, \gamma=4 / 3, \overline{\mathrm{b}}=0.05$

3. $\mathrm{M}_{\mathrm{A}}^{-2}=0.3, \gamma=4 / 3, \overline{\mathrm{b}}=0.1 ; 4 . \mathrm{M}_{\mathrm{A}}^{-2}=0.3, \gamma=5 / 3, \overline{\mathrm{b}}=0$; 0.1 ;

5. $\mathrm{M}_{\mathrm{A}}^{-2}=0.3, \gamma=5 / 3, \overline{\mathrm{b}}=0.05 ; 6 . \mathrm{M}_{\mathrm{A}}^{-2}=0.3, \gamma=5 / 3, \overline{\mathrm{b}}=$

7. $\mathrm{M}_{\mathrm{A}}^{-2}=0.35, \gamma=4 / 3, \overline{\mathrm{b}}=0$;

8. $\mathrm{M}_{\mathrm{A}}^{-2}=0.35, \gamma=4 / 3$,

$\overline{\mathrm{b}}=0.05$;

0

9. $\mathrm{M}_{\mathrm{A}}^{-2}=0.35, \gamma=4 / 3, \overline{\mathrm{b}}=0.1 ; 10 . \mathrm{M}_{\mathrm{A}}^{-2}=0.35, \gamma=5 / 3, \overline{\mathrm{b}}=$

11. $\mathrm{M}_{\mathrm{A}}^{-2}=0.35, \gamma=5 / 3, \overline{\mathrm{b}}=0.05 ; 12 . \mathrm{M}_{\mathrm{A}}^{-2}=0.35, \gamma=5 / 3$, $\overline{\mathrm{b}}=0.1$.

The effects of an increase in value of adiabatic exponent $\gamma$ are

(i) increases the value of $\beta$, i.e., decreases the shock strength (see Table 1);

(ii) to increase the pressure $P(\xi)$; whereas to decrease the flow variables $U(\xi), H(\xi), F(\xi)$ and $l_{\theta}$ at any point in the flow field behind the shock (see Figs. 1 (a, e, f-h));

(iii) the density $G(\xi)$ decreases near shock but increases near inner boundary surface (see Fig. 1(d)); (iv) the flow variables $\phi(\xi), V(\xi)$ and $l_{z}$ remain unaffected (see Fig. 1 (b, $\mathrm{c}, \mathrm{i})$ );

(v) increases the shock velocity $W / a_{1}$ (see Fig.2).

\section{CONCLUSION}

The similarity solutions for the propagation of magnetogasdynamics cylindrical shock waves in a rotational axisymmetric non-ideal gas with radiation heat flux have been obtained. The radiation heat flux and other flow variables in the flow field behind the shock have been evaluated from the equations of motion without using the radiation heat transfer equations explicitly. On the basis of this work, we may draw the following conclusions:

(i) The shock strength decreases and the shock velocity increases with an increase in the strength of the ambient magnetic field or the adiabatic exponent or the non-idealness of the gas.

(ii) An increase in the value of the parameter of nonidealness of the gas $\bar{b}$ decrease the radial component of fluid velocity, the axial magnetic field, the radiation flux and the azimuthal component of vorticity vector; whereas reverse behaviour is observed for the pressure. The density increases near shock but decreases near inner boundary surface with an increase in the value of parameter $\bar{b}$. Moreover, azimuthal, axial components of fluid velocity and axial component of vorticity vector remains unaffected with an increase in $\bar{b}$.

(iii) An increase in the strength of the ambient magnetic field $\mathrm{M}_{\mathrm{A}}^{-2}$ and value of parameter of non-idealness of the gas $\bar{b}$ have same behaviour on radial component of fluid velocity, density, the radiation flux and the azimuthal component of vorticity vector; whereas they have opposite behaviour for the pressure and the axial magnetic field.

(iv) An increase in the value of adiabatic exponent $\gamma$ and parameter of non-idealness of the gas $\bar{b}$ have same behavior on all the flow variables.

\section{REFERENCES}

[1] Chaturani P. (1970). Strong cylindrical shocks in a rotating gas. Appl. Sci. Res 23(1): 197-211. http://doi.org/10.1007/BF00413198

[2] Sakurai A. (1956). Propagation of spherical shock waves in stars. Fluid Mech 1(4): 436-453. http://doi.org/10.1017/S0022112056000275

[3] Nath O, Ojha S, Takhar HS. (1999). Propagation of a shock wave in a rotating interplanetary atmosphere with increasing energy. Mhd. Plasma Res 8(4): 269-282.

[4] Ganguly A, Jana M. (1998). Propagation of shock waves in a self-gravitating radiative magnetohydrodynamic non-uniform rotating atmosphere. Bull. Cal. Math. Soc 90: 77-82.

[5] Marshak RE. (1958). Effects of radiation on shock wave behavior. Phys. Fluids 1: 24-29. http://doi.org/10.1063/1.1724332

[6] Elliot LA. (1960). Similarity methods in radiation hydrodynamics. Proc. Roy. Soc 258(1294): 287-301. http://doi.org/10.2307/2413960

[7] Wang KC. (1964). The piston problem with thermal radiation. Fluid Mech 20(3): 447-455. http://doi.org/10.1017/S0022112064001343

[8] Taylor GI. (1950). The formation of a blast wave by a very intense explosion. I, II, Proc. Roy. Soc 201(1065): 159-174. http://doi.org/10.1098/rspa.1950.0049

[9] Ashraf S, Sachdev PL. (1970). An exact similarity solution in radiation-gas-dynamics. Proc. Indian Acd. Sci 71(6): 275-281. http://doi.org/10.1007/BF03049574

[10] Verma BG, Vishwakarma JP. (1978). An exact similarity solution for spherical shock wave in magnetoradiative gas. Astrophys. Space Sci 58(1): 139-147. http://doi.org/10.1007/BF00645381

[11] Anisimov SI, Spiner OM. (1972). Motion of an almost ideal gas in the presence of a strong point explosion. Appl. Math. Mech 36(5): 883-887. http://doi.org/10.1016/0021-8928(72)90144-X

[12] Rao MPR, Purohit NK. (1976). Self-similar piston problem in non-ideal gas. Int. J. Eng. Sci 14(1): 91-97. http://doi.org/10.1016/0020-7225(76)90059-8

[13] Vishwakarma JP, Nath G. (2007). Similarity solutions for the flow behind an exponential shock in a non-ideal gas, Meccanica 42(4): 331-339. http://doi.org/10.1007/s11012-007-9058-6

[14] Vishwakarma JP, Nath G. (2009). A self-similar solution of a shock propagation in a mixture of a non-ideal gas and small solid particles. Meccanica 44(3): 239-254. http://doi.org/10.1007/s11012-008-9166-y

[15] Nath G. (2012). Propagation of a cylindrical shock wave in a rotational axisymmetric isothermal flow of a nonideal gas in magnetogasdynamics. Ain Shams Eng 3(4): 393-401. http://doi.org/10.1016/j.asej.2012.03.009 
[16] Wu CC, Roberts PH. (1993). Shock-wave propagation in a sonoluminescing gas bubble, Phys. Rev. Lett 70(22): 3424-3427. http://doi.org/10.1103/PhysRevLett.70.3424

[17] Roberts PH, Wu CC. (1996). Structure and stability of a spherical implosion. Phys. Lett 213(1): 59-64. http://doi.org/10.1016/0375-9601(96)00082-5

[18] Levin VA, Skopina GA. (2004). Detonation wave propagation in rotational gas flows. Appl. Mech. Tech. Phy 45(4): 457-460. http://doi.org/10.1023/b:jamt.0000030320.77965.c1

[19] Nath G. (2011). Magnetogasdynamic shock wave generated by a moving piston in a rotational axisymmetric isothermal flow of perfect gas with variable density. Adv. Space Res, 47(9): 1463-1471. http://doi.org/10.1016/j.asr.2010.11.032

[20] Nath G. (2015). Similarity solutions for unsteady flow behind an exponential shock in an axisymmetric rotating non-ideal gas. Meccanica 50: 1701-1715. http://doi.org/10.1007/s11012-015-0115-2

[21] Vishwakarm JP, Patel N. (2015). Magnetogasdynamic cylindrical shock waves in a rotating nonideal gas with radiation heat flux. Eng. Phy. Thermophys 88(2): 521530. http://doi.org/10.1007/s10891-015-1217-3

[22] McVittie GC. Spherically solutions of the equations of gas dynamics. Proc. Roy. Soc 220(1142): 339-355. http://doi.org/ 10.1098/rspa.1953.0191

[23] Nicastro JR. (1970). Similarity analysis of radiative gasdynamics with spherical symmetry. Phys. Fluids, 13: 2000-2006. http://doi.org/10.1063/1.1693197

[24] Greifinger C, Cole D. (1961). On cylindrical magnetohydrodynamic shock waves. Phys. Fluids 4: 527-534. http://doi.org/10.1063/1.1706358

[25] Vishwakarma JP, Maurya AK, Singh KK. (2007). Selfsimilar adiabatic flow headed by a magnetogasdynamics cylindrical shock wave in a rotating non-ideal gas. Geophys. Astrophys. Fluid Dynamics 101(2): 155-168. http://doi.org/10.1080/03091920701298112

[26] Nath G, Vishwakarma JP. (2014). Similarity solution for the flow behind a shock wave in a non-ideal gas with heat conduction and radiation heat-flux in magnetogasdynamics. Commun. Nonlinear Sci. Numer. Simul 19(5): 1347-1365. https://doi.org/10.1016/j.cnsns.2013.09.009

[27] Vishwakarma JP, Shrivastava RC, Kumar A. (1987). An exact similarity solution in radiation magnetogasdynamics for the flows behind a spherical shock. Astrophys. Space Sci 129(1): 45-52. http://doi.org/10.1007/BF00717856

[28] Vishwakarma JP, Pandey SN. (2004). Magnetogasdynamic cylindrical shock waves in a nonideal gas with radiation heat flux. Modelling Meas. Control B 88(2): 23-37. http://doi.org/10.1007/s10891015-1217-3

[29] Rosenau P, Frankenthal S. (1976). Equatorial propagation of axisymmetric magnetohydrodynamic shocks. I. Phys. Fluids 19: 1889-1899. http://doi.org/10.1063/1.861424

[30] Pai SI. (1969). Inviscid flow of radiation gasdynamics (High temperature inviscid flow of ideal radiating gas, analyzing effects of radiation pressure and energy on flow field). Math. Phys. Sci 3: 361-370.

[31] Singh AK. (2012). Self-similar flow behind a cylindrical shock wave in a self-gravitating rotating gas with heat conduction and radiation heat flux. Modelling,
Measurment and Control B 81: 61-81.

[32] Vishwakarma JP, Maurya AK, Singh AK. (2011). Cylindrical shock waves in a non-ideal gas with radiation heat-flux and magnetic field. Modelling, Measurment and Control B 80(1/2): 35-52.

[33] Nath G, Dutta M, Pathak RP. (2017). An exact solution for the propagation of shock waves in self-gravitating medium in the presence of magnetic field and radiative heat flux Modelling. Measurement and Control B 86(4): 907-927.

http://iieta.org/sites/default/files/Journals/MMC/MMC B/86.04 06.pdf

\section{NOMENCLATURE}

\begin{tabular}{|c|c|}
\hline$a$ & function of $\mathrm{t}$ \\
\hline$a_{1}$ & constant \\
\hline$B$ & constant \\
\hline$b$ & $\begin{array}{l}\text { Van der Waals excluded } \\
\text { volume }\end{array}$ \\
\hline$b_{0}$ & function of $\mathrm{t}$ \\
\hline C & $\begin{array}{l}\text { angular velocity of the } \\
\text { medium }\end{array}$ \\
\hline$C_{a}$ & constant \\
\hline$C_{v}$ & $\begin{array}{l}\text { specific heat at constant } \\
\text { volume }\end{array}$ \\
\hline$c$ & function of $\mathrm{t}$ \\
\hline$d$ & constant \\
\hline$e$ & internal energy per unit mass \\
\hline $\bar{E}$ & $\begin{array}{l}\text { non-dimensional internal } \\
\text { energy per unit mass }\end{array}$ \\
\hline$E_{T}$ & $\begin{array}{l}\text { total energy of the flow-field } \\
\text { behind }\end{array}$ \\
\hline
\end{tabular}

shock front

F

$\bar{F}$

radiation flux

non-dimensional radiation flux

function of $\mathrm{t}$

non-dimensional density

function of $t$

non-dimensional azimuthal magnetic field

azimuthal magnetic field

constant

abbreviation

abbreviation

shock Mach number

Alfven- Mach number

function of $t$

non-dimensional fluid

pressure

pressure

fluid velocity

Shock radius

independent

coordinate

temperature of the gas

independent time coordinate

non-dimensional radial

component of fluid velocity 
$u$

V

$v$

$v^{*}$

W

W

$w_{a}$

$(r, \theta, z)$

\section{Greek Letters}

$\rho$

$\delta$

$\phi$

$\alpha$

$\beta$ radial component of fluid $\Gamma$

velocity

non-dimensional axial $\sigma$

component of fluid velocity

azimuthal component of fluid

velocity

the specific volume

shock velocity

axial component of fluid

velocity

constant

cylindrical coordinates

the fluid density

constant

non-dimensional azimuthal

component of fluid velocity

constant

ratio of density across the shock front

0 gas constant

ratio of specific heats

magnetic field variation

index.

magnetic permeability

arbitrary function of $\mathrm{r}$ and $\mathrm{t}$

constant

constant

similarity variable

vorticity vector

components of vorticity

vector

\section{Subscripts}

\section{Superscript}

immediately ahead the shock immediately behind the shock

derivative with respect to $t$ 